\title{
Datenschutz in der globalen Informationsgesellschaft
}

Ergebnisse der 30. Internationalen Konferenz der Datenschutzbeauftragten 2008 in Straßburg

„Wir leben in einer globalisierten Überwachungsgesellschaft und das weitgehend auch noch völlig freiwillig." Diese alarmierende Diagnose hat der Präsident des Bundesverfassungsgerichts, Prof. Papier, in seiner bemerkenswerten Rede anlässlich des 25jährigen Jahrestages des Volkszählungsurteils am 18. Dezember 2008 gestellt. In einer Welt grenzenloser Datenflüsse lässt sich Datenschutz nicht mehr als nationale Kirchturmpolitik betreiben.

Dieser an sich nicht neuen Erkenntnis stellten sich die Datenschutzbeauftragten bei ihrer 30. Internationalen Konferenz, zu der der deutsche Bundesbeauftragte für den Datenschutz und die Informationsfreiheit und die französische Commission $\mathrm{Na}$ tionale de l'Informatique et des Libertés (CNIL) anlässlich des dreißigjährigen Bestehens dieser beiden Datenschutzbehörden im Oktober 2008 nach Straßburg eingeladen hatten. Bereits in den zurückliegenden Jahren hatten die Datenschutzbeauftragten wiederholt die Notwendigkeit einer weltweiten Konvention zum Schutz der Privatsphäre hervorgehoben. In Straßburg unterstrichen sie in einer Entschließung erneut die Dringlichkeit international verbindlicher Normen zum Datenschutz.

Dabei konnten sie auf eine Reihe von zum Teil ermutigenden Entwicklungen hinweisen: Die Kommission für internationales Recht der Vereinten Nationen hat diesen Punkt in ihr Arbeitsprogramm aufgenommen. Der Europarat hat den Beitritt von Nichtmitgliedstaaten zur Datenschutzkonvention von 1981 befürwortet. Die OECD hat eine verstärkte grenzüberschreitende Zusammenarbeit bei der Anwendung von Rechtsvorschriften zum Schutz der Privatsphäre empfohlen. Auch regional sind verstärkte Initiativen für einen verbesserten Datenschutz zu beobachten: Nach der Europäischen Union entwickeln auch die Staaten in der asiatisch-pazifischen Region, in Iberoamerika und in der frankophonen Staatenfamilie, die viele afrikanische Länder einschließt, entsprechende, teilweise allerdings sehr heterogene Initiativen. Insgesamt bestehen erhebliche Disparitäten im Datenschutzniveau fort, für viele nationale Rechtsordnungen ist Datenschutz noch immer ein Fremdwort. Die Internationale Konferenz hat in Straßburg deshalb ihren Appell erneuert, ein zwingendes, universelles Rechtsinstrument zum Menschenrecht auf Schutz der Privatsphäre und der persönlichen Daten auszuarbeiten. Sie hat eine Arbeitsgruppe gebildet, die bis zur nächsten Konferenz im Herbst 2009 in Madrid einen gemeinsamen Vorschlag zur Erstellung internationaler Normen zum Schutz der Privatsphäre und zum Datenschutz erarbeiten soll. Dieses ehrgeizige Ziel wird angesichts der Vielzahl von Regelungsansätzen und der unterschiedlichen Rechtskulturen schwierig zu erreichen sein. Denn sowohl in den USA als auch in der asiatisch-pazifischen Region wird der Datenschutz im Gegensatz zu Europa bisher nicht als Grund- und Menschenrecht verstanden.

Der klassische regulatorische Ansatz zur Lösung von Datenschutzfragen stößt allerdings zunehmend an seine Grenzen. Weltweit setzt sich deshalb auch die Erkenntnis durch, dass gerade - aber nicht allein - für Kinder und Jugendliche praktische Maßnahmen ergriffen werden sollten, um ihnen bei der selbstverständlichen Nutzung des Internets und insbesondere der rasant wachsenden sozialen Netzwerke (social communities) Mittel zum Selbstschutz an die Hand zu geben. In zwei Entschließungen hat die Konferenz in Straßburg deshalb den Schutz der Privatsphäre von Kindern im Internet und den Datenschutz in sozialen Netzwerken unterstrichen. Den teilweise sittenwidrigen Marketing-Strategien gegenüber Kindern im Internet, die häufig mit massiver Ausforschung verbunden sind, muss auch mit verstärkten Anstrengungen zur Verbesserung der Medienkompetenz und entsprechenden Bildungsangeboten begegnet werden.

Die Entschließung zu sozialen Netzwerken geht zurück auf das Rom-Memorandum der Internationalen Arbeitsgruppe zum Datenschutz in der Telekommunika- tion, das im Frühjahr 2008 veröffentlicht wurde. Sie wendet sich mit konkreten Empfehlungen sowohl an die Nutzer als auch die Anbieter sozialer Netzwerkdienste. Während die Nutzer auf strikte Datensparsamkeit, die Verwendung von Pseudonymen und die Privatsphäre Anderer achten sollten, werden die Anbieter auf ihre besondere Verantwortung für die Belange der Nutzenden hingewiesen. Sie müssen bei grenzüberschreitenden Angeboten die Datenschutzstandards der Länder einhalten, in denen sie ihre Plattformen betreiben. Neben einer umfassenden Aufklärung der Nutzer muss deren Kontrolle über die Sichtbarkeit von Profilen und die Nutzung von Profil- und Verkehrsdaten weiter verbessert werden. Schon die Standardeinstellungen müssen datenschutzfreundlich sein, denn erfahrungsgemäß ändert nur eine Minderheit von Nutzern nach der Registrierung diese Einstellungen, weil sie sich nicht der von diesen Plattformen erzeugten Illusion der Intimität hingibt. Insbesondere bei Netzwerken, die sich an Minderjährige (z.B. Schüler) wenden, müssen diese Grundeinstellung besonders restriktiv sein. Die immer noch bestehenden Sicherheitsmängel der social communities müssen behoben werden. Auskunfts- und Löschungsrechte müssen ebenso gewährleistet werden wie die pseudonyme Nutzbarkeit von sozialen Netzwerken. Das massenhafte Herunterladen (bulk harvesting) von Nutzerdaten muss ebenso ausgeschlossen sein wie die Indexierung durch externe Suchmaschinen ohne ausdrückliche Zustimmung der Betroffenen.

Daneben hat die Internationale Konferenz, beschlossen, ihre Repräsentanz bei internationalen Organisationen zu intensivieren, einen eigenen Internet-Auftritt zu entwickeln und die Möglichkeit eines Internationalen Tages oder einer Woche für den Schutz der Privatsphäre nach dem Vorbild des 2007 vom Europarat initiierten Europäischen Datenschutztages zu prüfen.

\section{Alexander Dix, Berliner Beauftragter} für Datenschutz und Informationsfreiheit 